\title{
Prediction of therapeutical response to antidepressants based on pharmacological testing
}

\author{
T. Dakukina $^{1}$, T. Golubeva ${ }^{1}$, A. Gilep ${ }^{2}$, M. Machrov ${ }^{1}$, I. Haidukevich ${ }^{2}$.
}

${ }^{1}$ Republican scientific-practical center of mental health, Department of mental and behavioral disorders, Minsk, Belarus.

2 Institute of Bioorganic Chemistry, department of molecular biotechnologies, Minsk, Belarus.

\section{Objectives:}

In order to predict a therapeutic response to antidepressants in patients with pharmacoresistant depression, the presence of allelic variants of cytochrome CYP2C9, CYP2C19, CYP2D6 isoenzyme genes was determined. The results of pharmacogenetic testing were analyzed, and recommendations for the patient were developed taking into account the test results.

\section{Background:}

Problem of clinical psychiatry is optimizing the pharmacological treatment of depression. In the Republic of Belarus, the number of people with depression amounts to about $0.1 \%$ of the population. About $30 \%$ of patients don't respond to treatment. Individual genetic differences is the factor, which can cause the ineffectiveness of pharmacotherapy, development of adverse drug reactions.

\section{Materials and Methods:}

When patient identified slow allelic variants of CYP2C9*2, CYP2C9*3, CYP2C19*2, CYP2D6*4, it's recommended to choose drug that is not a substrate of the corresponding isoenzyme or adjust the dose because of the increased risk of side effects.

When fast allelic variant of CYP2C19*17 detected, it's recommended to choose a drug that is not a substrate of the corresponding isoenzyme, or to adjust the dose upwards due to a decrease in the effectiveness of therapy in dynamics.

\section{Results and Conclusions:}

Result of using this approach in the studied group of 102 depressed patients receiving treatment that corresponds to the clinical and genetic features of antidepressant metabolism, the need for re-hospitalization significantly decreased compared to the comparison group of 149 patients receiving treatment according to the clinical protocol features. In $63 \%$ of patients from the study group there was a significant reduction of depressive symptoms, improvement of social functioning when observed

Comparison between effectiveness of clinical response to antidepressants due to treatment based on clinical protocols $+P G x$ testing $(\boldsymbol{A})$ and only on clinical protocols (B)
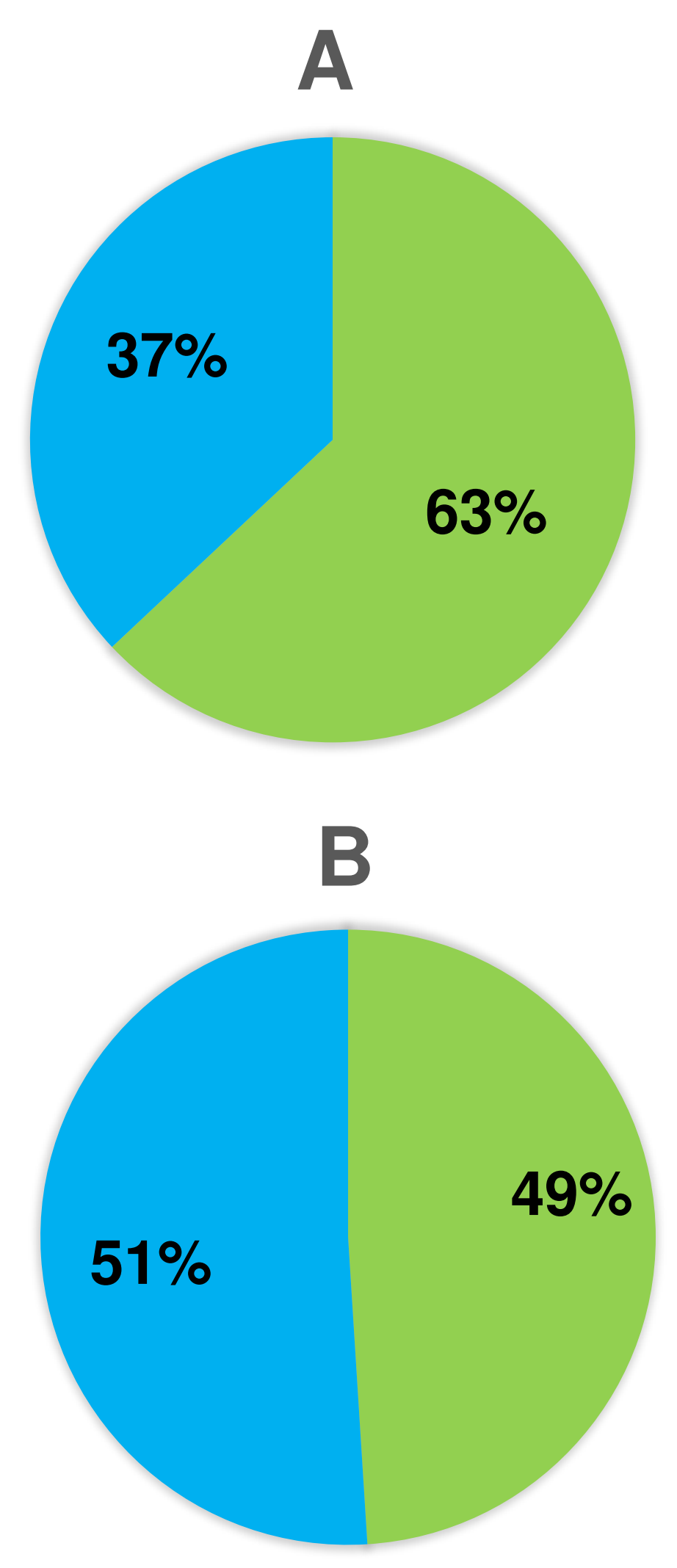

- Clinical improovement need for re-hospitalization 